\title{
Application of a picosecond soft $x$-ray source to time-resolved plasma dynamics
}

\author{
J. Workman, ${ }^{\text {a) }}$ M. Nantel, A. Maksimchuk, and D. Umstadter \\ Center for Ultrafast Optical Science, University of Michigan, Ann Arbor, Michigan 48109-2099
}

(Received 27 June 1996; accepted for publication 12 November 1996)

\begin{abstract}
We demonstrate the application of an ultrashort x-ray source as an external probe to measure plasma dynamics. The plasma is generated by a 100-fs Ti:sapphire laser focused onto thin metallic films. Time-resolved spectroscopy of the gold x-ray probe transmission through a perturbed $1000 \AA$ aluminum film reveals redshifts of the $L$-shell photoabsorption edge. We show that the dynamic behavior of this shift is consistent with the relaxation of the aluminum following the compression generated by a shock wave traveling through the film. An analytic plasma model, with comparison to a numerical hydrodynamics model, indicates compression up to 1.4 times solid density. (C) 1997 American Institute of Physics. [S0003-6951(97)00403-8]
\end{abstract}

A new class of x-ray sources has recently been introduced with the aid of high-intensity subpicosecond laser pulses, generated using the chirped pulse amplification technique. $^{1,2}$ Virtually no hydrodynamic expansion occurs during a subpicosecond laser interaction with a solid density target: what remains is a solid density plasma with up to $\mathrm{keV}$ electron temperatures. ${ }^{2-4}$ These conditions allow x-ray emission from high-lying ionization states that are short lived, due to the extremely large collision rates. Such x-ray sources are useful as diagnostic probes because of their high brightness, made possible by their short time duration and high densities. $^{2,5}$ Interest in these sources is wide spread owing to their potential applications, which include microlithography, time-resolved microscopy, diffraction, and spectroscopy studies of transient physical and chemical phenomena. ${ }^{6,7}$

Development and characterization of such X-ray sources have been ongoing. X-ray pulse duration measurements in the $1000 \mathrm{eV}$ spectral region have been accomplished using various target materials as well as various measurement techniques. ${ }^{2,5,8}$ The importance of laser intensity and contrast has been demonstrated for the generation of the shortest pulses as well as the broadest bandwidth $\mathrm{x}$-ray sources. ${ }^{4,5}$ Important applications of these sources, so far, have concentrated on the atomic physics of the plasmas from which the $\mathrm{x}$ rays originate. ${ }^{2,3}$

We present in this work what we believe to be the first use of such a source as a time-resolving probe in an application relevant to atomic, plasma, and high-energy-density matter physics. Using the x-ray source as a backlight, we adopt a pump-probe geometry to investigate the dynamic changes in electronic structure of a thin metallic film as it is perturbed by an ultrashort-laser pulse. Because the laser deposits its energy in a skin depth of about $100 \AA$ before expansion occurs, very large amplitude shock waves can be generated without material preheat commonly found in long pulse interactions. ${ }^{9}$ Under these novel conditions, predictions indicate the generation of up to gigabar pressure shock waves lasting picoseconds in duration. ${ }^{10,11}$ This raises the possibility of studying-high-energy density matter, relevant to inertial confinement fusion as well as astrophysical states of matter in small-scale laboratory experiments. In the past,

${ }^{a)}$ Electronic mail: workman@lanl.gov time-resolved measurements of $K$-edge shifts in plasmas driven by nanosecond pulses have been used to infer conditions in highly compressed materials. ${ }^{12,13}$ In this study, we use 100-fs laser pulses to impulsively drive shocks into our sample, measuring $L$-edge shifts, which are more accessible to soft $\mathrm{x}$-ray sources. The $10-\mathrm{Hz}$ repetition rate allows statistical averaging as well as clean signal-to-noise data. To gain an understanding of the experimental results, we use an analytic plasma model to describe the relationship between $L$-edge position and the associated plasma parameters. Reference to hydrodynamics simulation corroborates our understanding of the shock and relaxation dynamics.

The pump-probe measurements were made using a $100-\mathrm{fs} 10-\mathrm{Hz}$ Ti:sapphire laser system operating at a wavelength of $790 \mathrm{~nm}$. The $50 \mathrm{~mJ}$ laser pulses were sent to a beam splitter where $90 \%$ of the energy was reflected onto an f/5 off-axis parabola, used to focus the light to an intensity of $2 \times 10^{17} \mathrm{~W} / \mathrm{cm}^{2}$ onto a solid gold target. The broadband ultrashort-pulse $\mathrm{x}$-ray point source generated from this interaction was refocused to a sample at the opposite end of the experimental chamber using a section of a grazing incidence ellipsoidal optic; a central beam block eliminated any perturbations to the sample that might arise from hot electrons or hard $\mathrm{x}$ rays. The characteristics of the $\mathrm{x}$-ray probe are shown in Fig. 1. Broadband emission has previously been measured from 20 to $200 \AA, 4,5$ however, here we focus on the emission in the range of 100-200 $\AA$. The decrease in spectral intensity in Fig. 1 is due to the spectral response of the spectrometer grating and detectors. The pulse duration was limited to 20 ps due to intensity contrast limitations of the 790-nm laser light. While the pulse duration was measured with a jitterfree streak camera, ${ }^{14}$ the time-integrated spectrum in Fig. 1 was obtained with the microchannel plate (MCP) used for the pump-probe measurements. A calibrated x-ray diode, with filters transmitting photon energies at $100 \pm 50 \AA$, measured integrated soft $\mathrm{x}$-ray energies of $10-20 \mu \mathrm{J}$ at the source. Only a few tenths of a percent of this energy was collected by the ellipsoid, giving a total x-ray energy of approximately $50 \mathrm{~nJ}$ at the sample plane. The remaining $5 \mathrm{~mJ}$ of laser light was sent into an optical delay line and then focused with an $f / 10$ lens onto the thin metallic sample to an intensity of $1 \times 10^{14} \mathrm{~W} / \mathrm{cm}^{2}$. The sample plane was coincident with the slit of a variable spaced-grating grazing inci- 
(a)

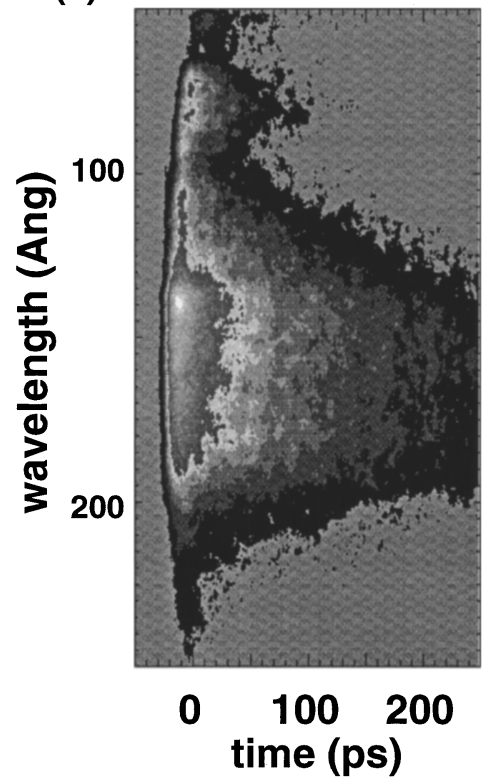

(b)

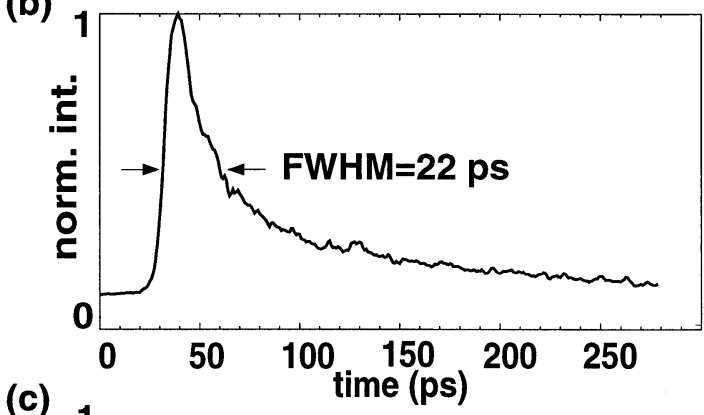

(c)

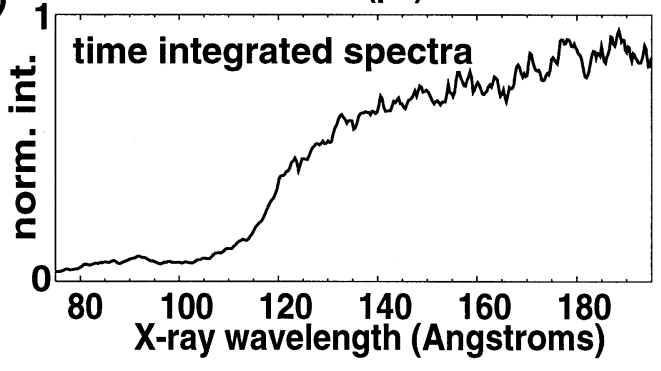

FIG. 1. Soft x-ray source generated by a 100-fs laser interaction with a solid gold target. (a) Temporally and spectrally resolved images of the source. (b) Temporal lineout near $170 \AA$ A. (c) The spectrum was measured separately with a microchannel plate.

dence imaging soft $\mathrm{x}$-ray spectrometer. The MCP coupled to the end of the soft x-ray spectrometer enabled us to record spectrally and spatially resolved $x$-ray absorption through the perturbed material at various delay times with respect to the laser pump. The sample consisted of $1000 \AA$ of aluminum deposited onto $2000 \AA$ of parylene- $n\left(\mathrm{C}-\mathrm{H}_{n}\right)$ as a rear-side supporting structure. Comparison of absorption spectra through cold aluminum to that of the perturbed aluminum was possible for each data point because the laser focus of $125 \mu \mathrm{m}$ was smaller than the $300-400 \mu \mathrm{m}$ x-ray probe focus.

The absorption feature used for analysis of the aluminum sample was the $L_{I I, I I I}$ photoabsorption edge at $72.78 \mathrm{eV}$ (170.04 ̊). ${ }^{15}$ The position of the edge gives a direct measure of the ionization potential and, thus, the electronic structure of the probed sample. The energy of the edge will change as a function of material density, temperature, and ionization state. $^{12,13}$ The measured shift in the $L_{\text {II,III }}$ edge is recorded as a function of probe delay in Fig. 2 for a pump-laser intensity of $1 \times 10^{14} \mathrm{~W} / \mathrm{cm}^{2}$. The early times $(t<0)$ correspond to probing the unperturbed sample with the soft $x$-ray source before the laser arrives. We then see a maximum redshift of $1.6 \pm 0.4 \AA$, which decreases at later times as the sample decompresses. The decompression occurs within approximately $50 \mathrm{ps}$, consistent with sound speed time scales through a cold $1000 \AA$ aluminum sample. Error bars indicate the time resolution of the 20-ps X-ray probe on the time axis and the averaging of several data points on the shift axis. Each data point consisted of 10-20 laser shots.

Hydrodynamic calculations of the 100-fs laser generated shock waves indicate that the shock will be generated in picoseconds and will traverse the $1000 \AA$ material on a time scale of the order 5-10 ps. ${ }^{10,16,17}$ This implies that our time resolution is ideal for measuring the dynamics of the relaxation of the material after the shock, but not yet sufficient to resolve the dynamics of the shock wave itself.
Analysis of the edge shift must address three physical processes in a purely plasma state model. These include ionization blueshifts, redshifts from continuum lowering, as well as edge shifts due to changes in the chemical potential (and, thus, the Fermi energy). Ionization blueshifts, $\Delta E_{\text {ion }}$, are calculated using Hartree-Fock theory while the StewartPyatt formalism is used to calculate redshifting due to continuum lowering, $\Delta E_{\mathrm{cl}}$. ${ }^{18}$ The effects of electron degeneracy on the chemical potential, $\Delta E_{\mathrm{deg}}$, are determined by an expansion around the Fermi energy. ${ }^{19}$ These three contributions sum together to give a total shift, $\Delta E_{\mathrm{tot}}=\Delta E_{\mathrm{ion}}+\Delta E_{\mathrm{cl}}$ $+\Delta E_{\mathrm{deg}} \cdot{ }^{12}$ Of course, in our geometry an absorption measurement integrates the contributions from each region of the sample, which can be roughly divided into a hightemperature low-density blow-off region, a high-density lowtemperature compressed region, and the undisturbed cold material. High-temperature regions contribute ionization

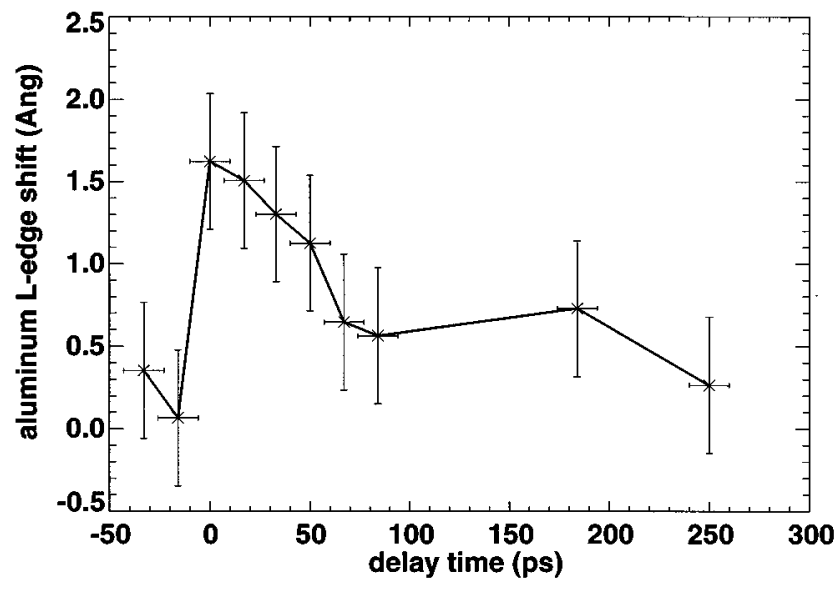

FIG. 2. Redshift of the aluminum $I_{\mathrm{II}, \mathrm{III}}$ edge as a function of x-ray probe delay time. 


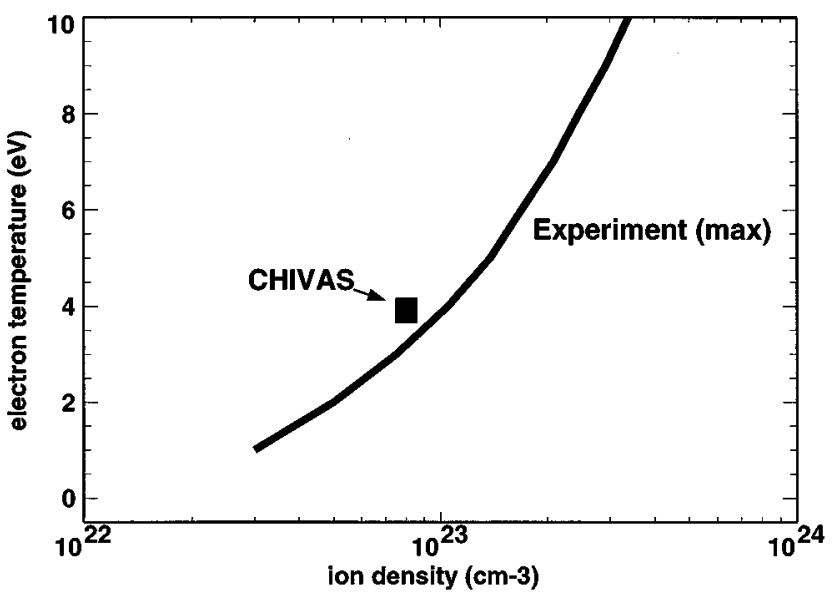

FIG. 3. Temperature and density parameter space spanned by the maximum observed edge shift (solid line) and the result of a 1.5-D hydrodynamic simulation (square box). Solid density for aluminum is $6 \times 10^{22} \mathrm{~cm}^{-3}$.

blueshifts that are masked by redshifts. We, therefore, probe the higher-density regions at any given time. In fact, we expect the measured shift to be a weighted average, resulting from contributions from different positions in the highdensity region.

Using the above plasma model, with Saha-based scaling for ionization as a function of temperature, we can plot the locus of points representing the maximum observed edge shift as a function of temperature and ion density. This parameter space is indicted in Fig. 3. The experimental error in the shift measurement is included in the width of the curve. This plot demonstrates the need for a measurement of the temperature to accurately determine the density, and hence, the compression in our sample. Without such a complimentary measurement, detailed hydrodynamic information is necessary to isolate a single set of parameters describing the plasma. With the use of the detailed 1.5-D hydrocode CHIVAS, we find a temperature and maximum compression that fall within our experimental boundaries. ${ }^{17}$ The numerical results show consistency with our analytic approach and indicate a maximum compression of 1.4 times solid density at a temperature of $3.9 \mathrm{eV}$. Analysis is ongoing.

We have successfully demonstrated the utility of these ultrashort-pulse $\mathrm{x}$-ray sources in an application that encompasses atomic physics, plasma physics, and high-energy density matter physics. Our preliminary analysis is consistent with an analytic model as well as with numerical simulation.
Further analysis should consider solid-state effects due to the low temperatures and high densities. Also, it is possible to reduce the time duration of the source to below $5 \mathrm{ps}$, which would allow us to resolve clearly the propagation of the shock wave in the sample. We expect that these short-pulse $\mathrm{x}$-ray probes will have a significant contribution to the understanding of ultrafast dynamics of this and other physical and chemical processes.

This work was supported by NSF Grant No. STC PHY 8920108, LLNL DOE Contract No. W-7405-ENG-48, and by the FCAR fund. The authors would like to thank G. Mourou, J.-C. Kieffer, and J.-C. Gauthier for useful discussions and A. Decoster for the use of the code CHIVAS, and Gabor Keiesar for integrated x-ray energy measurements. The authors also thank Spectrogon for the compressor gratings.

${ }^{1}$ G. Mourou and D. Umstadter, Phys. Fluids B 4, 2315 (1992).

${ }^{2}$ J. C. Kieffer, M. Chaker, J. P. Matte, H. Pepin, C. Y. Cote, Y. Beaudoin, T. W. Johnston, C. Y. Chien, S. Coe, G. Mourou, and O. Peyrusse, Phys. Fluids B 5, 2676 (1993).

${ }^{3}$ P. Audebert, J. P. Geindre, A. Rouse, F. Fallies, J. C. Gauthier, A. Mysyrowicz, G. Grillon, and A. Antonetti, J. Phys. B 27, 3303 (1994).

${ }^{4}$ M. M. Murnane, H. C. Kapteyn, M. D. Rosen, and R. W. Falcone, Science 251, 531 (1991).

${ }^{5}$ J. Workman, A. Maksimchuk, X. Liu, U. Ellenberger, J. S. Coe, C. Y. Chien, and D. Umstadter, Phys. Rev. Lett. 75, 2324 (1995).

${ }^{6}$ OSA Proceedings on Short Wavelength Coherent Radiation: Generation and Applications, 1991, edited by P. Bucksbaum and N. Ceglio (Optical Society of America, Washington, DC, 1991).

${ }^{7}$ C. P. Barty, F. Raksi, C. Rose-Petruck, K. J. Schafer, K. R. Wilson, V. V. Yakovlev, K. Yamakawa, Z. Jiang, A. Ikhlef, C. Y. Cote, and J. C. Kieffer, in Proceeding of the SPIE-The International Society for Optical Engineering 2521, 246 (1995).

${ }^{8}$ M. H. Sher, U. Mohideen, H. W. K. Tom, O. R. Wood II, G. D. Aumiller, R. R. Freeman, and T. J. McIlrath, Opt. Lett. 18, 646 (1993).

${ }^{9}$ Ya. B. Zel'dovich and Yu. P. Raizer, Physics of Shock Waves and HighTemperature Hydrodynamic Phenomena (Academic, New York, 1967).

${ }^{10}$ V. E. Gusev, Phys. Vibr. 57, 1 (1993).

${ }^{11}$ A. Ng, A. Forsman, and P. Celliers, Phys. Rev. E 51, R5208 (1995).

${ }^{12}$ D. K. Bradley, J. Kilkenny, S. Rose, and J. D. Hares, Phys. Rev. Lett. 59, 2995 (1987).

${ }^{13}$ L. DaSilva, A. Ng, B. K. Godwal, G. Chiu, and F. Cottet, Phys. Rev. Lett. 62, 1623 (1989).

${ }^{14}$ A. Maksimchuk, M. Kim, J. Workman, G. Korn, J. Squire, D. Du, D. Umstadter, G. Mourou, and M. Bouvier, Rev. Sci. Instrum. 67, 697 (1996).

${ }^{15}$ B. L. Henke, P. Lee, T. J. Tanaka, R. L. Shimabukuro, and B. K. Fujikawa, J. Opt. Soc. Am. B 3, 1540 (1986).

${ }^{16}$ J. C. Gauthier (private communication).

${ }^{17}$ S. Jacquemot and A. Decoster (private communication).

${ }^{18}$ J. C. Stewart and K. D. Pyatt, Jr., Apl. J. 144, 1203 (1966).

${ }^{19}$ E. Kiess, Am. J. Phys. 55, 1006 (1987). 